\title{
The Entangled Dance of Atom Beams
}

\section{Entangled pairs of ultracold atoms interfere in a double waveguide.}

\author{
By Rachel Berkowitz
}

$\square$ hysically distant quantum particles can share properties and can behave as a single object. For pairs of photons, this entangled behavior allows for data transfer between the two particles, for example. Quantum entanglement can also occur between more massive particles, including two individual atoms. Now, in a controlled experiment, Filippo Borselli of the Vienna University of Technology, Austria, and colleagues create traveling beams of entangled atoms that are reliably produced from a reservoir source using two parallel waveguides [1]. The experiment could be a starting point for developing integrated quantum circuits and quantum-information processing devices that use pairs of atoms.

One way to produce pairs of correlated atoms is by delivering a single quantum of vibrational energy to every atom in a Bose-Einstein condensate (BEC) - a low-density gas of ultracold atoms-which then loses that surplus energy by emitting pairs of atoms. The Vienna group previously leveraged this behavior, exciting a BEC on a tiny chip, called an atom chip, that traps atoms just above the chip's surface. They found their chip

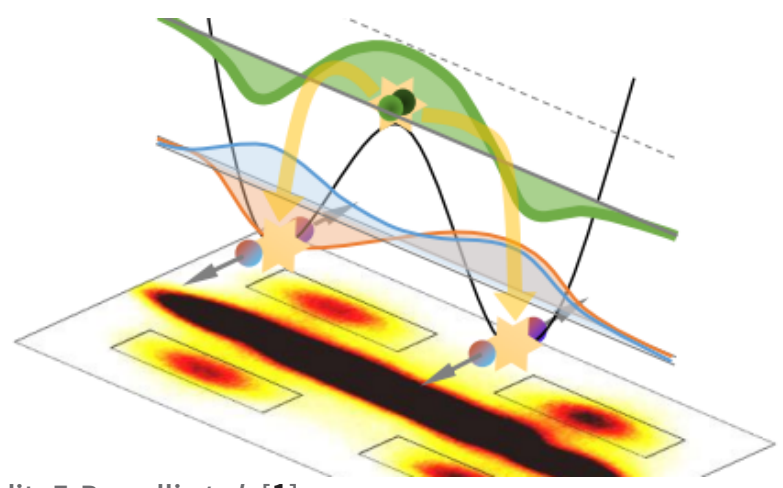

Credit: F. Borselli et al. [1] emitted pairs of atoms that propagated in opposite directions because of conservation of momentum.

Building on that work, the team modified their atom chip so that it included two parallel waveguides that were positioned to capture the emitted atoms. The chip was designed so that the atoms could travel into either the left or the right waveguide. By measuring the momentum correlations between the atoms in the pairs after they had passed through the waveguide and observing the fringe pattern produced by interference between the two possible quantum paths, the team deduced that the particles behaved as a single quantum object.

Rachel Berkowitz is a Corresponding Editor for Physics based in Vancouver, Canada.

\section{REFERENCES}

1. F. Borselli et al., "Two-particle interference with double twin-atom beams," Phys. Rev. Lett. 126, 083603 (2021). 